\title{
A Community-Level Perspective on Digitally and Socially Including Older People
}

\author{
James Richardson \\ Good Things Foundation \\ 1 East Parade, Sheffield, UK, S1 2ET \\ jamesrichardson@goodthingsfoundation.org
}

\begin{abstract}
A presentation of findings from the evaluation of the Future Digital Inclusion programme funded by the UK Government Department of Education. Data show the effectiveness of embedding digital inclusion support within wider social inclusion services at the local level, mediated by institutions and organisations which have established pathways to engage older people.
\end{abstract}

Keywords: digital exclusion, social exclusion, digital inclusion, older people, adult and community learning

\section{INTRODUCTION}

There are still 11.3 million people in the UK who don't have the full range of digital skills 'needed to safely benefit from, participate in and contribute to the digital world of today and the future', including $10 \%$ of the workforce. ${ }^{1,2}$ The people lacking them tend to be older, have fewer qualifications, earn less or have disabilities: the same groups who are already experiencing other forms of social exclusion, which is exacerbated by their exclusion from the huge benefits digital technology can offer. $^{3,4}$ In the context of assistive technology, it is clear that this skills gap needs to be addressed in order to ensure that the potential benefits can be realised by everyone. For the last 3 years, the UK Government-funded Future Digital Inclusion programme has been supporting digitally excluded people to improve their digital skills, confidence and access.

\section{THE FDI PROGRAMME}

Funded by the UK Government Department for Education, the Future Digital Inclusion (FDI) programme aims to teach Basic Digital Skills to 1 million digitally excluded people. ${ }^{5}$ Delivery is coordinated by a national charity (Good Things Foundation) and delivered by a network of independent community-level organisations (the Online Centres network) offering free or low cost access to the internet, alongside support to help people develop the skills they need. Although grant funding is disbursed to some of these organisations to support some digital inclusion (DI) activities, a much larger number deliver DI without FDI grant funding using a range of resources created and curated by Good Things Foundation, including an online learning platform and free marketing materials. Delivery organisations tend to offer additional support services tailored to the needs of geographic or local demographic communities, which may include but are not limited to employment support, benefits advice, financial capability training, ESOL (English for Speakers of Other Languages), basic literacy and numeracy skills, and accredited qualifications. In addition, they operate a support model which is flexible (with skills training tailored to individual needs) and informal (with an emphasis on peer-to-peer learning, volunteer support, and socialisation).

By providing DI infrastructure support to the community sector, and incorporating digital inclusion into existing social inclusion activities, the FDI programme creates considerable economies of scope and scale, with measurable improvements in a range of social inclusion indicators. This includes a range of digital skills, but also employment and volunteering, further learning, and various measures of wellbeing. Above all, the programme has been able to engage socially excluded people, often facing complex barriers, who are not engaged with alternative provision such as that offered by Further Education colleges.

\section{METHODS}

Evaluation of the Future Digital Inclusion programme used a range of methodological tools and approaches to corroborate data and gain a clear understanding of the relationship between learners' needs, Online Centre support and the contribution of Good Things Foundation. These methods include: 
i. A detailed Social Return On Investment (SROI) analysis of Future Digital Inclusion grant-funded Online Centres, carried out by Just Economics. ${ }^{6}$ This SROI analysis calculates the value of Future Digital Inclusion activities to individuals, communities and the state.

ii. A second independent report by Just Economics, examining the effect of network membership on Online Centres, especially in terms of support provided in addition to grant funding, and the ways in which Online Centres support learners to progress from learning Basic Digital Skills to Further Education.

iii. An independent report by the Centre for Economic and Business Research (Cebr) into the economic benefit of a $100 \%$ digitally skilled population, based on the FDI-funded Online Centres support model. ${ }^{7}$

iv. The creation of and engagement with a 'Research Partner Network', a subset of selected active and engaged Online Centres to provide ongoing insight into the circumstances facing different kinds of Online Centre and demographics, and support the delivery of the 'Routes to Inclusion' study (below).

v. The user-centred 'Routes to Inclusion' Longitudinal Learner Study, carried out through the Research Partner Network, recruited 29 learners from a variety of backgrounds for 18 months. Analysis of audio-recorded interviews focussed on motivations and triggers for learning; personal circumstances, especially in terms of barriers to achievement and inclusion; and the nature of the support provided by Online Centres. This userfocused activity has collected completely original qualitative data on the interaction between digital and social exclusion.

vi. Learner baseline and progression surveys which are independently administered by IFF Research. Surveys are administered online at baseline (following registration with an online learning platform, Learn My Way) to capture learners' motivations, circumstances and demographics; and three months afterwards via telephone, to capture changes in motivation, skills and access, as well as progression to further learning and employment. For the period of the evaluation, 4,770 people completed the baseline survey and 620 completed the subsequent progression survey.

vii. A quarterly survey of Online Centres (administered by Good Things Foundation) to capture data on trends in centre capacity as a result of Future Digital Inclusion funding including Basic Digital Skills for organisations (Go ON UK 2014 matrix), facilitating a deep level of quantitative and qualitative analysis regarding outcomes.

viii. Conducting an audit of the Online Centres Network which has contributed to a more detailed segmentation of the network in terms of delivery models, activities and target audiences. This included a number of quantitative surveys administered online and by telephone by IFF Research, as well as qualitative data gathering through visits and phone calls by Good Things Foundation.

\section{RESULTS}

Demographics and outcomes for people trained were monitored by Good Things Foundation through an ongoing survey of learners administered by independent research agency IFF, and data collected automatically through the Learn My Way platform. The table below summarises learner demographic and progression data for 2017-18, highlighting some of the hard-to-reach groups the programme has supported.

Table 1: Demographics of FDI Learner Population

\begin{tabular}{|c|c|}
\hline Demographic & $\%$ of FDI Learners \\
\hline Unemployed & 32 \\
\hline Aged over 55 & 31 \\
\hline Disabled $^{8}$ & 25 \\
\hline Educated $<$ Level $2^{9}$ & 48 \\
\hline HBAl income poverty ${ }^{10}$ & 27 \\
\hline Social housing tenants & 37 \\
\hline Means-tested benefits & 60 \\
\hline
\end{tabular}

The support provided by Online Centres has resulted in improvements in Future Digital Inclusion learners' skills, motivation and personal circumstances, measured by progression to, towards or within employment (62\%), to further learning $(83 \%)$, and to using online government services (70\%). Other observed learner progression includes:

- $41 \%$ of learners accessed health information online for the first time; a further $32 \%$, who already accessed health information online, learned to do so more effectively

- $54 \%$ of learners would as a first resort access online resources such as NHS.UK for non-urgent medical advice 
- $89 \%$ of unemployed learners undertake activity that helps them move towards employment, including online job search and creating online CVs.

$88 \%$ of learners with low skills undertook further learning, half of whom started studying towards a qualification.

\section{DISCUSSION}

Older people stand to gain a great deal from the use of digital technology, including assistive technology, mobile applications and web-based resources. Data from the FDI programme clearly show that, at point of engagement, older learners were more likely to have negative attitudes towards digital technology, but that they benefited greatly from the support offered through the FDI model. These attitudes and outcomes are especially significant among older learners with low educational attainment.

$45 \%$ of learners over 55 reported that they had not enjoyed learning at school, compared to only $29 \%$ of the wider learner population; $79 \%$ report that they enjoy learning more as a result of the support they received at an Online Centre, compared to $57 \%$ of the wider population. Learners over 55 with lower education were the least likely to have personal access to the internet through any device (16\% vs $33 \%$ ), the least likely to have a favourable view of their internet skills (66\% vs $83 \%)$, and more likely to limit their online activity to one or two familiar websites (51\% vs $35 \%)$. They were also less likely to have engaged in any education or training in the three years prior to their engagement (57\% vs $71 \%)$.

The motivations of older learners reflect their lifestage and socioeconomic situation. Many older offline people rely on family proxies to carry out online tasks on their behalf, and loss of proxy support appears to motivate them to go online: only $13 \%$ of Online Centres learners give as their reason for learning that 'someone used to do online tasks on my behalf but now I have to do them for myself'; for over 65s the figure is more than double, at $28 \%$. $^{11}$ Learners were significantly more interested at point of engagement with using the internet to keep in touch with family and friends (69\% vs $49 \%$ younger learners), to find health information $(41 \%$ vs $35 \%)$ and to access government services (44\% vs $37 \%$ ). Older learners were also more likely to use their Online Centre for social contact (28\% vs $17 \%$ younger learners).

Progression for older learners through FDI reflects these motivations: they were more likely than learners under 55 to have used the internet for the first time for informal communication and social media ( $43 \%$ vs $21 \%$ ), and to have used online sources of public information and public service portals (56\% vs $12 \%$ ). Critically, they were more likely to report improved wellbeing as a result of face-to-face social contact at their Online Centre ( $76 \%$ vs $44 \%$ ), and to say that they felt less lonely and isolated through the opportunity to undertake community learning ( $83 \%$ vs $35 \%$ ). They were also more likely to feel that the social aspect of learning was an important contributor to their increased digital skills and confidence ( $75 \%$ vs $23 \%$, although the lower percentage among younger learners may be because many are unemployed and are required to attend Online Centres under direction of their Jobcentre). These results reflect the critical importance of informal, social contact for successful engagement and success in digital learning.

\section{CONCLUSIONS}

FDI is a Government-funded DI delivery initiative; although it has a strong research element, it is not only or principally designed to only collect data on digital inclusion practice, but rather to implement a programme of DI support, and measure impact as accurately as possible. The amount of resource that could be dedicated from the budget to support evaluation activities is not comparable to an academic research budget, making some goals like true randomisation of survey sampling - difficult to achieve. To compensate for these limitations, researchers at Good Things Foundation used a broad range of data collection methods and crossreferenced different data sources to compensate as much as possible for sampling errors or biases. Researchers made it clear when presenting their findings that these errors existed.

Despite these methodological limitations, there is still strong evidence that FDI helps people to become digitally included by embedding DI practice in existing support services for socially excluded people, delivered by organisations operating at a community level. These organisations have the clearly demonstrated capacity to engage people who could benefit from assistive technology and, more widely, digital health self-management tools; use of these tools is widely taught by the Online Centres network. Since FDI provision tends to be tailored to individual needs rather than following a set curriculum, there is scope to embed more specialist training on specific pieces of adaptive technology, which could be included as part of a wider programme of structured support. With increasing interest in such initiatives from the NHS at a national and regional level, partnerships between academics, tech innovators and DI practitioners should be developed that ensure assistive technology can be used by everyone who needs it.

These findings demonstrate the importance of incorporating DI into wider socially inclusive activities, which has not so far been adequately 
addressed in academic and grey literature which tends to place emphasis on developing skills and specific types of usage divorced from context and other needs. This is original research which bridges the gap between DI research and other forms of research into social interventions. There is strong relevance to the field of assistive technology research in general and AAATE in particular, in terms of the DI needs of older people, who need to develop digital skills and confidence before they are able to use many forms of assistive technology effectively.

\section{REFERENCES}

1 Lloyds Bank (2018). Lloyds Bank UK Consumer Digital Index 2018. Retrieved May 11 2018, from:

https://www.lloydsbank.com/assets/media/pdfs/b anking_with_us/whats-happening/LB-ConsumerDigital-Index-2018-Report.pdf

2 The Tech Partnership (2018). Essential Digital Skills Framework 2018. Retrieved May 11 2018, from: https://www.thetechpartnership.com/wpcontent/uploads/2018/05/EssentialDigitalSkillsFr amework-11May18.pdf

3 Dutton, W.H. and Blank, G., with Groselj, D. (2013). Cultures of the Internet: The Internet in Britain. Oxford Internet Survey 2013. Oxford Internet Institute, University of Oxford.

4 Ofcom. (2016). Adults' media use and attitudes. Retrieved February 24, 2017, from https://www.ofcom.org.uk/_data/assets/pdf_file/ 0026/80828/2016-adults-media-use-andattitudes.pdf?lang=uqovrjuc

5 Go ON UK. (n.d.). Basic Digital Skills framework. Retrieved February 24, 2017, from https://goonuk-prod.s3-eu-west-

1.amazonaws.com/uploads/Basic-Digital-Skills-

Framework-FINAL.pdf

6 Just Economics. (2016). A Social Return on Investment Analysis for Tinder Foundation. Retrieved February 24, 2017, from:

https://www.goodthingsfoundation.org/sites/default/ files/research-

publications/sroi250216formatted4_0.pdf

7 Cebr. (2015). The economic impact of Basic Digital Skills and inclusion in the UK. Retrieved February 24, 2017, from:

https://doteveryone-prod.s3-eu-west1.amazonaws.com/uploads/The\%20economic\% 20impact\%20of\%20digital\%20skills\%20and\%20i nclusion\%20in\%20the\%20UK_Final_23_11_15. pdf
8 UK Government. (2010). Equality Act 2010, Section 6. Retrieved February 24, 2017, from http://www.legislation.gov.uk/ukpga/2010/15/sect ion $/ 6$

9 UK Government Department for Education. (n.d). Retrieved February 24, 2017, from https://www.gov.uk/what-different-qualificationlevels-mean/list-of-qualification-levels

10 UK Government Depart for Work and Pensions. (2013). Retrieved February 24, 2017, from https://www.gov.uk/government/collections/hous eholds-below-average-income-hbai--2

11 William H. Dutton and Grant Blank, with assistance from Darja Groselj (2013): Cultures of the Internet: The Internet in Britain. Oxford Internet Survey 2013 Report, Oxford Internet Institute. Retrieved May 11 2018, from: http://oxis.oii.ox.ac.uk/wpcontent/uploads/sites/43/2014/11/OxIS-2013.pdf 